\title{
Unternehmensentwicklung in der OÖG Holding
}

\section{Gemeinsam für Generationen - Nachhaltig für Generationen Vernetzt - abgestuft - regional - subsidiär}

\author{
Mit Gründung der Oberösterreichischen Gesundheitsholding \\ $\mathrm{GmbH}(\mathrm{OÖG})$ und ihrer Beteiligung am Kepler Universitäts- \\ klinikum entstand ein Konzern, der mit einem Marktanteil \\ von $54,5 \%$ der größte Gesundheitsdienstleister in \\ Oberösterreich ist. Insgesamt beschäftigt die OÖG \\ ca.14.500 Mitarbeiterinnen und Mitarbeiter.
}

Angelika Edtstadler

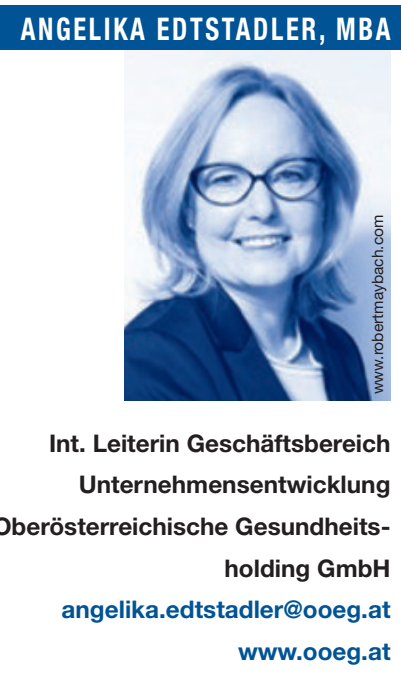

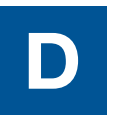
ie Ausrichtung des Konzerns muss so geplant werden, dass der erweiterten Mission entsprochen werden kann, die neben aktuellen Herausforderungen im operativen Geschehen auch die universitäre Weiterentwicklung des Kepler Universitätsklinikums (KUK) berücksichtigt.

Ein Unternehmen dieser Größe benötigt klare Strukturen und abgestimmte Prozesse, die einerseits die Leistungserbringung auf höchstem Qualitätsniveau gewährleisten und andererseits die zukünftige gemeinsame Ausrichtung aller Standorte sowie die nachhaltige Weiterentwicklung des Gesamtkonzerns ermöglichen.

\section{Der Geschäftsbereich Unternehmensentwicklung (UE)}

Im Herbst 2019 wurde der Geschäftsbereich „Unternehmensentwicklung“ unter der Leitung von Mag. Dr. Franz Harnoncourt, Vorsitzender der Geschäftsführung, eingerichtet und Abb.1 veranschaulicht dessen Organisationsstruktur. Die Aufgabenbereiche umfassen Strategie, interne Unternehmensberatung, Multiprojektmanagement, strategische Organisationsentwicklung neuer Bereiche sowie Evaluierung und Steuerung.

Schon am Beginn wurde deutlich, dass eine erfolgreiche Unternehmensentwicklung weder zentral noch Top-down erfolgen kann, sondern nur durch Einbindung der für die unterschiedlichen Bereiche verantwortlichen Führungskräfte und Schlüsselmitarbeiter/innen Erfolg haben kann. Dabei spielt das - noch im Aufbau befindliche - konzernweite Unternehmensentwicklungs-Netzwerk eine zentrale Rolle. „Networking“ bedeutet im Geschäftsbereich vor allem Austausch, Vertrauen, Offenheit, Zwanglosigkeit und die Bereit- 
Abb. 1: Organigramm Geschäftsbereich UE

๑ OOEG

\section{Oberösterreichische Gesundheitsholding GmbH (OÖG) \\ Unternehmensentwicklung}

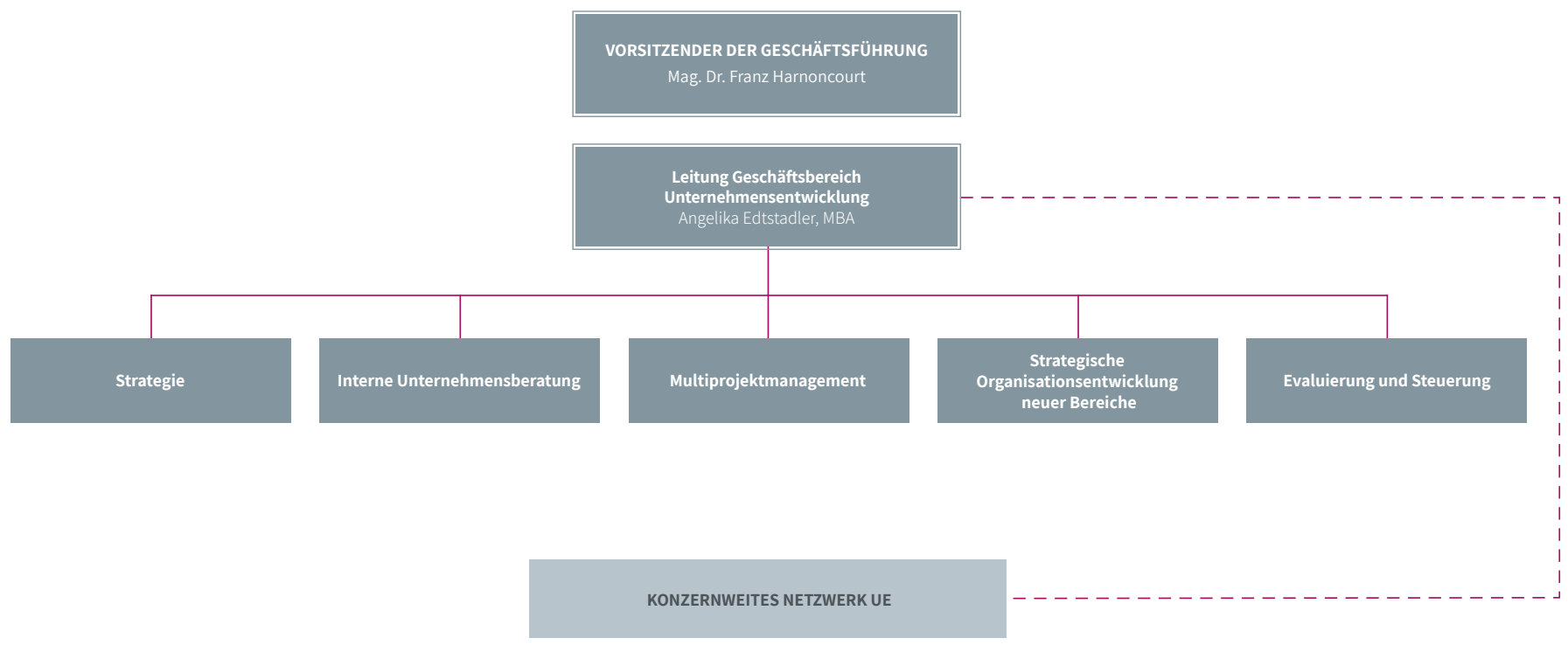

schaft, gemeinsam Botschafter für das Unternehmen OÖG sein zu wollen.

Ein weiteres Ziel des Geschäftsbereiches besteht darin, die bereits bestehenden Entwicklungsstrukturen und die vorhandenen strategischen Festlegungen der Gesundheits- und Spitals-AG (Gespag) und des Kepler Universitätsklinikums (KUK) bestmöglich zu nutzen. Die Gesamtausrichtung wird durch eine am neuen Kontext orientierte Analyse schrittweise transformiert.

\section{Säulen der Unternehmensentwicklung}

Die drei großen Säulen des Geschäftsbereiches UE beziehen sich auf die strategische Vernetzung auf unterschiedlichen Ebenen, die Entwicklung und Planung von Strategieprozessen sowie die Darstellung und Beurteilung der Ergebnisse (Abb. 2).
Im Jahr 2020 wurde verstärkt in die Bereiche „Aufbau - Strukturen - Vernetzung“ und „Strategieentwicklung" investiert, mit dem vorrangigen Ziel, neue Strukturen einzuführen und Konzernstrategien einzugleisen.

\section{Vorgehen bei der Strategieentwicklung}

Zahlreiche Voranalysen bildeten die Grundlage für diese wichtige Strategiearbeit nach dem Zusammenschluss.

Dabei nahmen die 17 Nachhaltigkeitsziele der Vereinten Nationen (Sustainable Development Goals, SDGs) und die weltweiten Megatrends einen zentralen Stellenwert ein. Sie bilden die Eckpunkte der strategischen Positionierung des Konzerns, wobei bei den Nachhaltigkeitszielen der Schwerpunkt der Weiterentwicklung auf die sozialen (SDG 3 - Gesundheit und Wohlergehen, SDG 4 - Hochwertige Bildung, SDG 5 - Geschlechtergleichheit, SDG 8 - Menschenwürdige Arbeit und Wirtschaftswachstum) und ökologischen Nachhaltigkeitsziele (SDG 7 - bezahlbare und saubere Energie, SDG 13 - Nachhaltige Energiegewinnung und Klimaschutz) gelegt werden wird.

In Bezug auf Megatrends konzentriert sich der Konzern vor allem auf zukunftsweisende Entwicklungen in den Bereichen Digitalisierung und Wissensmanagement.

Die Ausrichtung der Strategie orientiert sich an den relevanten Interessengruppen der Organisation. Daher nahm die Analyse der Interessengruppen und die Priorisierung nach ihrer Bedeutung für das Unternehmen einen zentralen Stellenwert ein. Darüber hinaus wurden die internen Stärken und Schwächen untersucht sowie externe Chancen und Risiken abgewogen. 
Abb. 2: Säulenmodell Geschäftsbereich UE

(c) Edtstadler

\begin{tabular}{|c|c|c|}
\hline $\begin{array}{l}\text { AUFBAU - STRUKTUREN - } \\
\text { VERNETZUNG }\end{array}$ & $\begin{array}{l}\text { STRATEGIE- } \\
\text { ENTWICKLUNG }\end{array}$ & $\begin{array}{l}\text { ZIELE UND } \\
\text { ERGEBNISSE }\end{array}$ \\
\hline Verortung des GB im KUK & Vorgehensmodell entwickeln & \multirow{2}{*}{$\begin{array}{l}\text { Definition Wertschöpfungs- } \\
\text { kette } \\
\text { Strategiereview - KUK }\end{array}$} \\
\hline $\begin{array}{l}\text { Mitarbeiter/innen und Netz- } \\
\text { werk aufbauen }\end{array}$ & $\begin{array}{l}\text { Zeitliche Planung des Ent- } \\
\text { wicklungsprozesses }\end{array}$ & \\
\hline $\begin{array}{l}\text { Verantwortlichkeiten/ } \\
\text { Zuständigkeiten vereinbaren }\end{array}$ & $\begin{array}{l}\text { Strategieentwicklungs- } \\
\text { prozess einführen }\end{array}$ & $\begin{array}{l}\text { Strategiereview - Regional- } \\
\text { kliniken }\end{array}$ \\
\hline Einsatz von Tools abstimmen & \multirow{2}{*}{$\begin{array}{l}\text { Coaching des übergeordne- } \\
\text { ten Strategie-Entwicklungs- } \\
\text { prozesses }\end{array}$} & \multirow{2}{*}{$\begin{array}{l}\text { Vorgehen zur konzernweiten } \\
\text { Abstimmung der Ergebnisse } \\
\text { erarbeiten }\end{array}$} \\
\hline $\begin{array}{l}\text { Festlegung von Kommunika- } \\
\text { tionsstrukturen }\end{array}$ & & \\
\hline \multirow{4}{*}{$\begin{array}{l}\text { Planung der Vorgehensweise } \\
\text { im Multiprojektmanagement } \\
\text { Aufbau Interessenpartner- } \\
\text { netzwerk }\end{array}$} & $\begin{array}{l}\text { Abstimmung und Kommuni- } \\
\text { kation der Ergebnisse }\end{array}$ & \multirow{2}{*}{$\begin{array}{l}\text { Planung der Darstellung } \\
\text { der Ergebnisse in einem GF } \\
\text { Dashboard } \\
\text { Multiprojektmanagement } \\
\text { etablieren }\end{array}$} \\
\hline & $\begin{array}{l}\text { Einbindung vorhandener } \\
\text { Strategien in die Gesamt- } \\
\text { ausrichtung }\end{array}$ & \\
\hline & $\begin{array}{l}\text { Festlegung der übergeordne- } \\
\text { ten Konzernstrategie }\end{array}$ & \multirow[t]{2}{*}{$\begin{array}{l}\text { Nachverfolgung der Beiträge } \\
\text { zur Strategieerreichung }\end{array}$} \\
\hline & $\begin{array}{l}\text { Planung Strategie- } \\
\text { weiterentwicklung }\end{array}$ & \\
\hline
\end{tabular}

Ein Hauptaugenmerk wurde auf die Betrachtung und grafische Aufbereitung der veränderten internen und externen Umwelten gelegt, die sich durch die Einbindung des Kepleruniversitätsklinikums in die OÖG ergaben. Die nachfolgende Darstellung des Konzernumfeldes, aufgebaut um das Logo der OÖG, das einem DNA-Strang nachempfunden ist, soll den Mitarbeitern/innen bildlich veranschaulichen, dass ihr Wirken stark abhängig ist von internen/externen Einflüssen und Anforderungen - dem sogenannten „ECO System" "1 einer Organisation.

Basierend auf der Mission des Unternehmens, den vorhandenen Werten der OÖG und des Kepler Universitätsklinikums wurde zunächst die Vision des Konzerns formuliert und die strategische Ausrichtung abgestimmt. Im Zuge dessen konnte festgelegt werden, dass sich die übergeordnete Ausrichtung an den Mitarbeitern/innen, am Kernprozess, an den Finanzen, an der IT und an der Kommunikation orientiert. Eine strategische Ausrichtung an Strukturen und Organisationseinheiten wurde aufgrund der Größe und Komplexität des Unternehmens bewusst vermieden.

In mehreren Vorstellungsrunden erfolgten Einladungen der kollegialen Führungen der unterschiedlichen Kliniken zum „Sounding“. Die Rückmeldungen enthiel- ten wichtige Informationen, die in die Strategieentwicklung eingearbeitet werden konnten. Ende 2020 wurde die Ausrichtung des Konzerns dem Aufsichtsrat der OÖG vorgestellt.

\section{Weiterentwicklungsplanung}

Auf Basis der übergeordneten Ausrichtung der OÖG geht es im heurigen Jahr 2021 in die Detailstrategieentwicklung bzw. in den Strategiereview am Kepleruniversitätsklinikum.

Bei der Realisierung der strategischen Ausrichtung setzt die Unternehmensentwicklung auf das strategische Multipro- 


\section{Abb. 3: ECO-System OÖG Holding}

๑ OOEG

\section{ECO - DNA - OÖG}

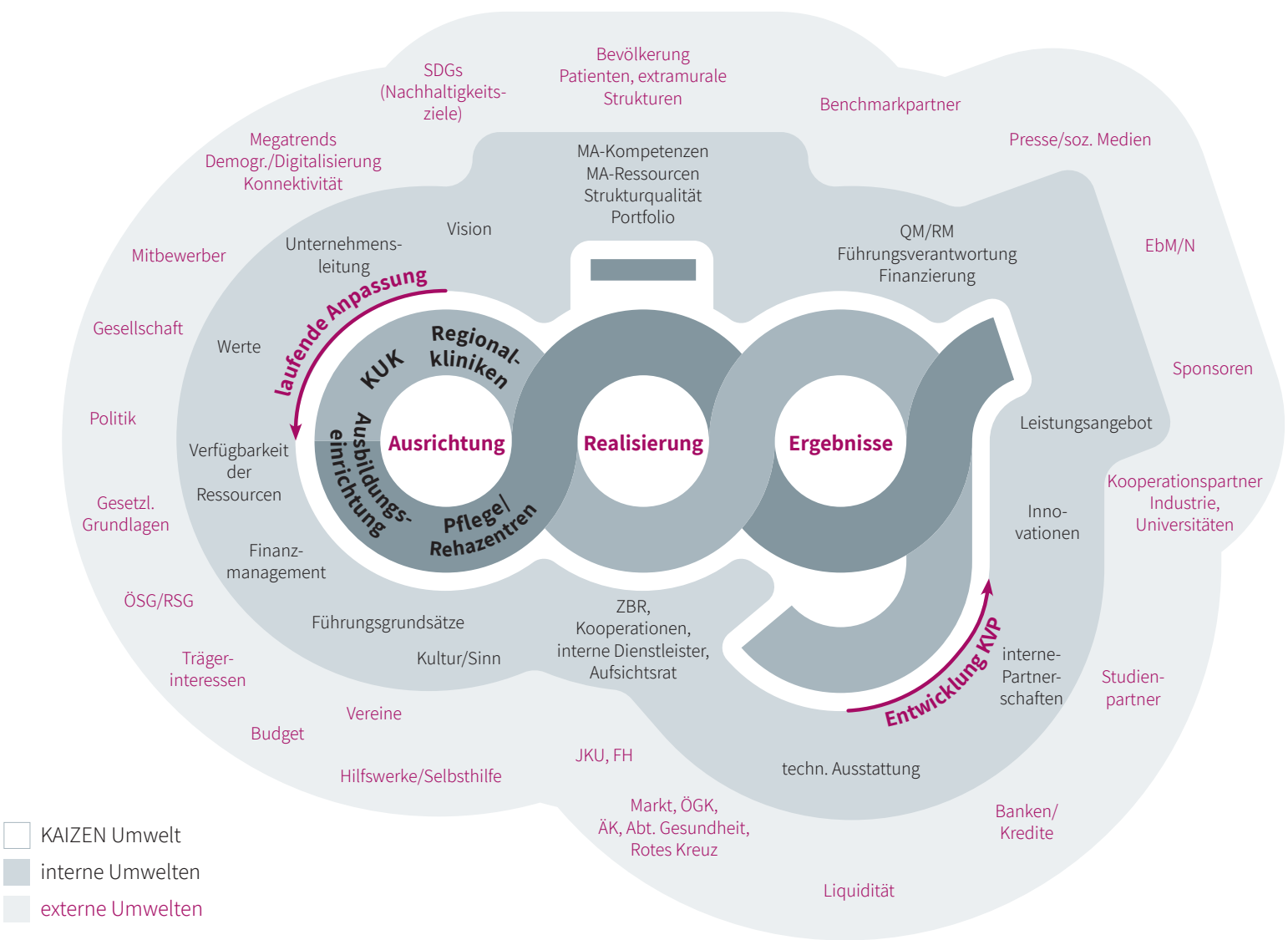

jektmanagement mittels Programmen, die zur Bewertung der Strategieerreichung beitragen werden (vgl. Angelika Edtstadler, Kepler Universitätsklinikum - „zusammen_wachsen" mit Programm, Qualitas $1 / 2016)^{2}$.

Die Ergebnisdarstellung orientiert sich an den wesentlichen Zielen und wird so gestaltet, dass steuerungsrelevante Kennzahlen der Geschäftsführung einen ganzheitlichen Überblick über die Weiterentwicklung des Konzerns entlang der übergeordneten Ausrichtung geben.

\section{Zusammenfassung}

Die strategische Ausrichtung der OÖG ist ein fortlaufender Prozess, der so angelegt ist, dass auf ungeplante Ereignisse, weltweite Trends, Verknappung von Ressourcen etc. flexibel reagiert werden kann. Damit wir diese Flexibilität in der zukünftigen Entwicklung gewährleisten können, ist es notwendig, dass das „Kerngeschäft“ an den verschiedenen Standorten stabil, sicher, mit höchster Qualität und möglichst ungestört erbracht werden kann. Dies zeigt sich bei der COVID-19-Pandemie aktuell mit großer Deutlichkeit: Nur ein stabiles Schiff wird in stürmischer See sein Ziel erreichen.
Literatur:

1 Quality Austria (Hrsg.):

Das EFQM Modell (deutsch) - Version 2020, ISBN: 978-90-5236-846-7.

2 Autoren: Angelika Edtstadler „Klinisches Programm Management Kepler Universitätsklinikum", in: Qualitas 2/2016)

- Burkhard Görtz, Silke Schönert, Kim Norman Thiebus: Programm-Management: Großprojekte planen, steuern und kontrollieren. München: Hanser Verlag, 2012.

- Project Management Institute (Hrsg.): The Standard for Program Management, $3^{\text {rd }}$ Edition. Project Management Institute, Inc., 2013. 